\title{
Synthesis of $\alpha$-diazoketones by the Action of Diazo-n-octane on 3-methoxy Cinnamoyl Chloride
}

\author{
DEVENDRA KUMAR GANGWAR ${ }^{\star}$ and A.K. AGARWAL \\ Department of Chemistry, Bareilly College, Bareilly - 253 005, India. \\ *Corresponding author E-mail : gangwardevendra2@ gmail.com
}

http://dx.doi.org/10.13005/ojc/320345

(Received: March 07, 2016; Accepted: April 17, 2016)

\begin{abstract}
The reaction of 3-methoxy cinnamoyl chloride $(1 \mathrm{~mol})$ with diazo-n-octane $(2 \mathrm{~mol}$ and 3 mol) gives 1-diazo-1-n-heptyl-4-(3-methoxy) phenyl but 3-ene 2-one and 4-(3-methoxy) phenyl3-n-heptyl 1-diazoacetyl-5-n-heptyl pyrazoline. The diazoketones were characterised by various physico-chemical techniques.
\end{abstract}

Keywords: Diazo-n-octane, 3-methoxy cinnamoyl chloride.

\section{INTRODUCTION}

A number of methods for synthesis of $\alpha$-diazo ketones by the reaction of higher diazoalkanes with carboxylic acid chlorides or acid anhydrides ${ }^{1-4}$. The field of synthesis of new $\alpha$-diazoketones is too wide ${ }^{5-7}$. The study of literature shows that a very little work has been done with higher diazo alkanes ${ }^{8-10}$. By using different amount of diazoalkane, it is possible to attack one or both the sites present in it. By doing so, it is possible to compare the reactivity of these sites ${ }^{11}$. In most cases the acid chloride ${ }^{12}$ group is attacked first and the other side afterwards. Thus starting from 3-methoxy cinnamoyl chloride $(1 \mathrm{~mol})$ and diazo-n-octane (2 $\mathrm{mol}$ and $3 \mathrm{~mol}$ ) 1-diazo-1-n- heptyl-4-(3-methoxy) phenyl 3-ene-2-one and 4-(3-methoxy) phenyl-3- n-heptyl 1-diazoacetyl-5-n-heptyl pyrazoline were synthesized following the method of Arndt-Eistert.

Above diazoketones were light yellow viscous liquids. The easily removal diazo group present in them, prevented their purification by distillation even under vaccum.

\section{EXPERIMENTAL}

1. Synthesis of 1-diazo-1-n- heptyl-4-(3methoxy) phenyl 3-ene-2-one : It was prepared by using 3-methoxy cinnamoyl chloride $(2.8 \mathrm{~g}, 1 \mathrm{~mol})$ on pre-estimated diazon-octane $(3.99 \mathrm{~g}, 2 \mathrm{~mol})$ at $0^{\circ} \mathrm{C}$. The reaction mixture was then kept at $0^{\circ} \mathrm{C}$ overnight. On removal of ether at low temperature the 
diazoketone was obtained as yellow mobile liquid which contained nitrogen.

The diazoketone so obtained was characterised by elemental analyses and its reactions with 2,4-dinitrophenyl hydrazine, benzoic acid, phenol and dry hydrochloric acid.

2. Synthesis of 4-(3-methoxy) phenyl-3-n-heptyl 1-diazoacetyl-5-n-heptyl pyrazoline : It was prepared by using 3-methoxy cinnamoyl chloride $(2.8 \mathrm{~g}, 1 \mathrm{~mol})$ on pre-estimated diazo$\mathrm{n}$-octane $(5.98 \mathrm{~g}, 3 \mathrm{~mol})$ at $0^{\circ} \mathrm{C}$. The reaction mixture was then kept at $0^{\circ} \mathrm{C}$ overnight. On removal of ether at low temperature the diazoketone was obtained as yellow mobile liquid which contained nitrogen.

\section{$\left(\mathrm{OCH}_{3}\right) \mathrm{C}_{6} \mathrm{H}_{4}-\mathrm{CH}=\mathrm{CH}-\mathrm{COCl}+2 \mathrm{C}_{6} \mathrm{H}_{13}-\mathrm{CH}_{2}-\mathrm{CHN}_{2}$ 3-Methoxy Cinnamoyl \\ diazo-n-octane} Chloride

$$
\left(\mathrm{OCH}_{3}\right) \mathrm{C}_{6} \mathrm{H}_{4}-\mathrm{CH}=\mathrm{CH}-\mathrm{CO}-\underset{\mathrm{n}-\mathrm{CH}_{2}-\mathrm{C}_{6} \mathrm{H}_{13}}{\mathrm{CN}_{2}}+\mathrm{C}_{6} \mathrm{H}_{13}-\mathrm{CH}_{2}-\mathrm{CH}_{2} \mathrm{Cl}+\mathrm{N}_{2}
$$

\section{$\left(\mathrm{OCH}_{3}\right) \mathrm{C}_{6} \mathrm{H}_{4}-\mathrm{CH}=\mathrm{CH}-\mathrm{COCl}+3 \mathrm{C}_{6} \mathrm{H}_{13}-\mathrm{CH}_{2}-\mathrm{CHN}_{2}$ \\ 3-Methoxy Cinnamoyl \\ diazo-n-octane} Chloride

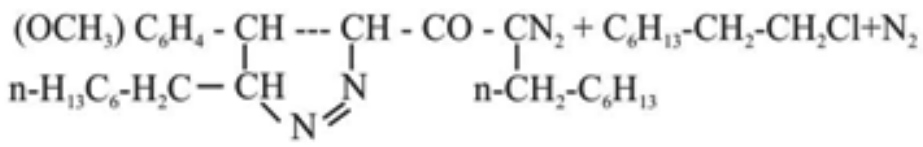

$$
\left(\mathrm{OCH}_{3}\right) \mathrm{C}_{6} \mathrm{H}_{4}-\mathrm{CH}=\mathrm{CH}-\mathrm{CO}-\underset{{ }_{\mathrm{n}}-\mathrm{CH}_{2}-\mathrm{C}_{6} \mathrm{H}_{13}}{\mathrm{C}_{2}}
$$

1-diazo-1-n- heptyl-4-(3-methoxy) phenyl 3-ene-2-one

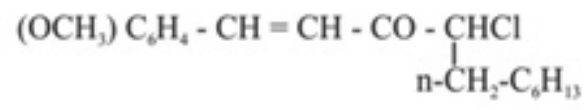

1-chloro-1-n- heptyl-4-(3-methoxy) phenyl 3-ene-2-one (chloro ketone)

$$
\left(\mathrm{OCH}_{3}\right) \mathrm{C}_{6} \mathrm{H}_{4}-\mathrm{CH}=\mathrm{CH}-\mathrm{CO}-\underset{\mathrm{I}}{\mathrm{C}} \mathrm{CHOCOC}_{2}-\mathrm{C}_{6} \mathrm{H}_{33}
$$

1-benzoyloxy-1-n- heptyl-4-(3-methoxy) phenyl 3-ene-2-one (ester)

$$
\begin{array}{r}
\left(\mathrm{OCH}_{3}\right) \mathrm{C}_{6} \mathrm{H}_{4}-\mathrm{CH}=\mathrm{CH}-\mathrm{CO}-\mathrm{CHOC}_{6} \mathrm{H}_{5} \\
\text { n- }-\mathrm{CH}_{2}-\mathrm{C}_{6} \mathrm{H}_{13}
\end{array}
$$

1-phenyloxy-1-n- heptyl-4-(3-methoxy) phenyl 3-ene-2-one (ether)

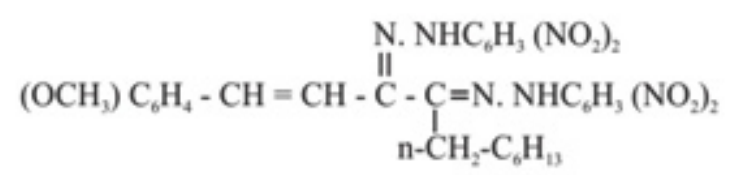

2,4-dinitrophenyl osazone

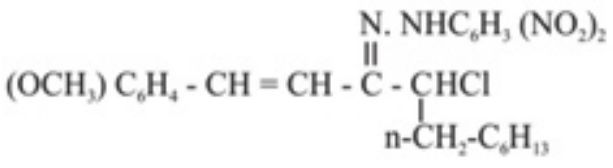

2,4-dinitrophenyl hydrazone<smiles>CCCC(OC(=O)OCc1ccccc1)C(C=CC(C)OCCOC)NCC</smiles>

2,4-dinitrophenyl hydrazone<smiles>CCCCC(C=CC(CC)OCCOC)C(=NCCC)O[N+](=O)[O-]</smiles>

2,4-dinitrophenyl hydrazone 
The diazoketone so obtained was characterised by elemental analyses and its reactions with 2,4-dinitrophenyl hydrazine, benzoic acid, phenol and dry hydrochloric acid.

The elemental analyses and IR spectral studies were carried out at CDRI Lucknow.

\section{RESULTS AND DISCUSSION}

Characterisation of 1-diazo-1-n- heptyl-4-(3methoxy) phenyl 3-ene-2-one

The diazoketone with an aqueous alcoholic sulphuric acid solution of 2, 4- dinitrophenyl hydrazine gave a 2,4-dinitrophenyl osazone as an orange solid, which after crystallisation from ethanol, melted at $158^{\circ} \mathrm{C}$ (Found $\mathrm{C}=55.78 \%, \mathrm{H}=4.55 \%, \mathrm{~N}=17.32 \%$, $\mathrm{C}_{30} \mathrm{H}_{32} \mathrm{O}_{9} \mathrm{~N}_{8}$, required $\mathrm{C}=55.55 \%, \mathrm{H}=4.93 \%$, $\mathrm{N}=17.28 \%$ ) absorbed frequencies $\mathrm{IR}(\mathrm{KBr}): 3435$ $(-\mathrm{NH}), 1632(\mathrm{C}=\mathrm{N}), 1620\left(-\mathrm{C}_{6} \mathrm{H}_{5}\right), 1355\left(\mathrm{C}-\mathrm{NO}_{2}\right), 970(-$ $\mathrm{CH}=\mathrm{CH}-), 724 \mathrm{Cm}^{-1}\left(\mathrm{CH}_{2}\right.$ rock in $\left.-\mathrm{C}_{7} \mathrm{H}_{15}\right)$. With dry $\mathrm{HCl}$ formed chloroketone afforded 2, 4- dinitrophenyl hydrazone. With benzoic acid gave an ester, afforded 2,4 nitro phenyl hydrazone. (Found $\mathrm{C}=63.12 \%, \mathrm{H}=$ $5.32 \%, \mathrm{~N}=9.25 \%, \mathrm{C}_{31} \mathrm{H}_{34} \mathrm{O}_{7} \mathrm{~N}_{4}$, required $\mathrm{C}=64.80 \%$,
$\mathrm{H}=5.92 \%, \mathrm{~N}=9.75 \%)$ absorbed frequencies $\mathrm{IR}(\mathrm{KBr})$ : $3350(-\mathrm{NH}), 1720(\mathrm{C}=\mathrm{O}), 1615(\mathrm{C}=\mathrm{N}), 1580\left(-\mathrm{C}_{6} \mathrm{H}_{5}\right)$, 1320(C- $\left.\mathrm{NO}_{2}\right), 965(-\mathrm{CH}=\mathrm{CH}-), 724 \mathrm{Cm}^{-1}\left(\mathrm{CH}_{2}\right.$ rock in- $\mathrm{C}_{7} \mathrm{H}_{15}$ ). With phenol gave an ether, afforded 2,4 nitro phenyl hydrazone.

\section{Characterisation of 4-(3-methoxy) phenyl-3-n-} heptyl 1-diazoacetyl-5-n-heptyl pyrazoline

The diazoketone with an aqueous alcoholic sulphuric acid solution of 2, 4- dinitrophenyl hydrazine gave a 2,4-dinitrophenyl osazone as an orange solid, which after crystallisation from ethanol melted at $132^{\circ} \mathrm{C}$ (Found $\mathrm{C}=57.76 \%, \mathrm{H}=6.20 \%$, $\mathrm{N}=17.33 \%, \mathrm{C}_{38} \mathrm{H}_{48} \mathrm{O}_{9} \mathrm{~N}_{10}$, required $\mathrm{C}=57.86 \%$, $\mathrm{H}=6.09 \%, \mathrm{~N}=17.56 \%)$ absorbed frequencies $\mathrm{IR}(\mathrm{KBr})$ : $3440(-\mathrm{NH}), 1632(\mathrm{C}=\mathrm{N}), 1620\left(-\mathrm{C}_{6} \mathrm{H}_{5}\right)$, 1332 $\left(\mathrm{C}-\mathrm{NO}_{2}\right), 725 \mathrm{Cm}^{-1}\left(\mathrm{CH}_{2}\right.$ rock in $\left.-\mathrm{C}_{7} \mathrm{H}_{15}\right)$. With dry $\mathrm{HCl}$ formed chloroketone afforded 2, 4- dinitrophenyl hydrazone. With benzoic acid gave an ester, afforded 2,4 nitro phenyl hydrazone. (Found $\mathrm{C}=65.14 \%, \mathrm{H}=$ $7.25 \%, \mathrm{~N}=11.12 \%, \mathrm{C}_{39} \mathrm{H}_{50} \mathrm{O}_{7} \mathrm{~N}_{6}$, required $\mathrm{C}=65.54 \%$, $\mathrm{H}=7.00 \%, \mathrm{~N}=11.76 \%)$ absorbed frequencies $\mathrm{IR}(\mathrm{KBr})$ : $3350(-\mathrm{NH}), 1720(\mathrm{C}=\mathrm{O}), 1625(\mathrm{C}=\mathrm{N}), 1590$ $\left(-\mathrm{C}_{6} \mathrm{H}_{5}\right), 1320\left(\mathrm{C}-\mathrm{NO}_{2}\right), 724 \mathrm{Cm}^{-1}\left(\mathrm{CH}_{2}\right.$ rock in $\left.-\mathrm{C}_{7} \mathrm{H}_{15}\right)$. With phenol gave an ether, afforded 2,4 nitro phenyl hydrazone.

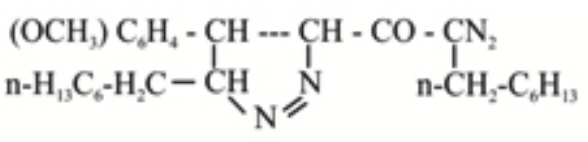
4-(3-methoxy) phenyl-3-n-heptyl 1-diazoacetyl-5-n-heptyl pyrazoline

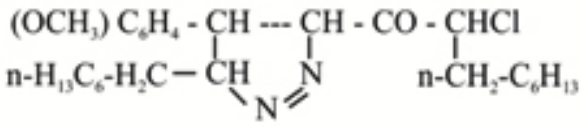

4-(3-methoxy) phenyl-3-n-heptyl 1-chloro-5-n-heptyl pyrazoline (chloro ketone)

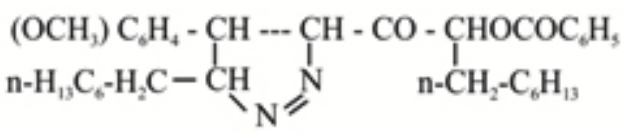
4-(3-methoxy) phenyl-3-n-heptyl 1-benzoyloxy-5-n-heptyl pyrazoline (ester)

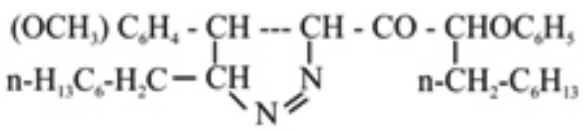

4-(3-methoxy) phenyl-3-n-heptyl 1-phenyloxy-5-n-heptyl pyrazoline (ether)

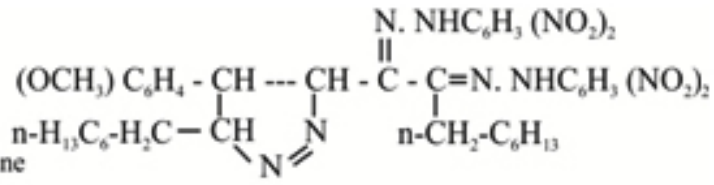

2,4-dinitrophenyl osazone

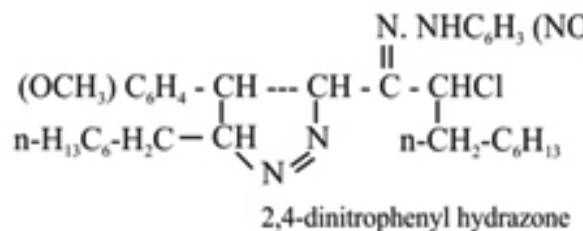

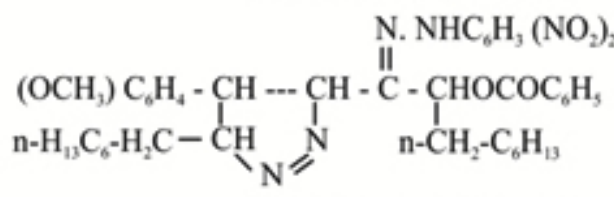

2,4-dinitrophenyl hydrazone<smiles>CCCCC1N=NC(C(=NCCC)C(CC)O[N+](=O)[O-])C1CCOC</smiles>

2,4-dinitrophenyl hydrazone 


\section{ACKNOWLEDGEMENT}

The authors are thankful to the principal, Bareilly College, Bareilly and Head, Department of
Chemistry for providing necessary facilities and also CD.R.I, Lucknow for various infra red spectra and nitrogen estimations.

\section{REFERENCES}

1. Sudrik, S.G.; Sharma J.; Chavan. V.B.; Chaki, N.K. Sonwane, H.R.; Vijaymohanan K.P. Org. Lett. 2006, 8, 1089.

2. Toma, T., Shimokawa, J., Fukuyama, T., Org. Lett., 2007, 9, 3195-3197.

3. Yadav J.S., Reddy, B.V.S., Rao Y.G. Narsalah A.V. Tetrahedron Lett.2008 49, 2381.

4. Taber D.F.; Tian, W.; J. Org. Chem; 2007, 72, 3207-3210.

5. Ceasar J., Dolenc, S., Tetrahedron Lett., 2001, 42, 7099-7102.

6. Pace. V.; Verniest, G.; Sinisterra, V; Akantara, A.R.; Kimbe, N.D., J. Org. Chem 2010, 75, 5760-5763.
7. Chakraborti S.; Agarwal, A.K.; Orient J. Chem. 2010, 26, 1573-1575.

8. Arndt F., Eistert B., Partale W., Ber. 1927, 60, 1364.

9. Gupta S.; Agarwal, A.K.; Garg, P.; Asian J. Chem, 2010, 22, 2939-2942.

10. Saxena. N.; Agarwal, A.K.; Chandra, A.; Imam, S.G.; Orient J. Chem. 2013, 29, 11931195.

11. Gupta, S., Garg, P., Agarwal, A.K., Orient J. Chem., 2009, 25, 457-458.

12. Saxena N.; Chandra A.; Imam, S.G.; Chandra, G.; Orient J. Chem., 2013, 29, 1085-1088. 